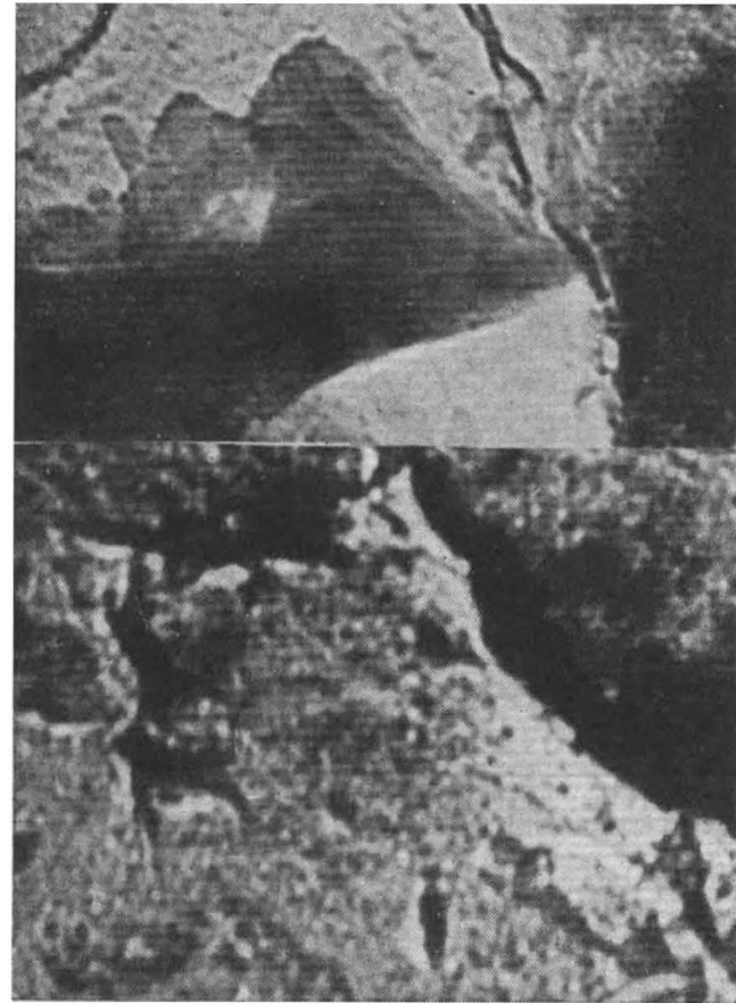

Fig. 5. MontmormLonite. The layer is so thin that the structure system is visible, though in three dimensions. Upper picture, $\times 80,000$; lower picture, $\times 40,000$

placed one upon the other, but with a slight displacement of each in relation to the one beneath it, in a manner similar to that observed in kaolinite, and form piles, sometimes resembling threads. In a fracture of montmorillonite perpendicular to the natural stratification, these piles appear raised. Sometimes, especially when the layer of the clay removed is very thin (Method 1), figures of a regular form are to be seen in the fracture, indicating that the small crystals or plates may have regular positions in the direction of stratification. In this case it is possible that the plates are arranged in hexagonal nets, often not regular, as may be seen in some photographs, forming channels or cavities in the montmorillonite, The above-mentioned piles of plates, showing slight undulations, constitute the walls of these cavities.

The peeled-off thin layer of clay, bentonite, etc. (Method 1), has been produced on fractures perpendicular to, as well as on fractures parallel to, the natural direction of stratification.

If the montmorillonite is built up in the way suggested, that is to say, of piles of crystals with a comparatively slight force of adhesion between them, it will split into thin, flat pieces, somewhat like scales, whenever the preparation is made (Method 1) on a fracture perpendicular to that of stratification. The $C$-axis of the small crystals will then be parallel to the plane of the photograph. If the 'scales' are thin enough, the piles will be fairly clearly visible. Sometimes the 'scales' do not remain plane, their outer parts turning up and showing a very marked propensity to absorb electrons, but this effect may also depend on a variation in the thickness of the scales. In this particular the aspect is very similar to that observed when bentonite is suspended in water.

In the preparation of the clay surface and the removal of the cellulose acetate film, some of the 'plates' in the piles would appear to accompany one side of the fracture and some the other. The consequence is that line structures appear in the casts, these being holes left by withdrawn 'plates'. The thickness of the lines generally shows that they must represent two or more atomic layers together with the water-layers lying between. Occasionally, how. ever, lines are observed that cannot be estimated as exceeding $15 \mathrm{~A}$. in thickness. These suggest the cavities left by single atomic layers, with their attendant water-layers, withdrawn from the pile. That such narrow lines can be observed with such sharpness is due to the fact that the objects reproduced are layers seen edgewise, or cavities between such layers, with a depth of absorption some ten times greater than the width of the lines. The method should be capable of further development as a means of determining the resolving power of the electron microscope.

\section{ATMOSPHERIC OSCILLATIONS*}

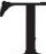

HE theory of the tides is one of the many offshoots of Newton's theories of mechanics and of gravita. tion. Much of the later development of these theories was achieved by the great French school of mathematicians, and the first important steps in the theory of the atmospheric tides were made by Laplace (1774) and later described in his "Mécanique Céleste". $\mathrm{He}$ also was the first to attempt to determine the lunar atmospheric tide from barometric observations; he realized that tropical data were the most suitable for the purpose, but lacking them, he used 4,752 readings at Brest. His result, as he recognized, did not give the true tidal pressure variation at Brest, which is too small to bè separated, in such a short series of data, from the solar daily variations and the much larger irregular changes associated with the weather.

\section{The Lunar Atmospheric Tide}

The lunar atmospheric tide was first determined in 1842 by Lefroy from bihourly barometric readings over seventeen months at the British Colonial Magnetic and Meteorological Observatory at St. Helena $\left(16^{\circ} \mathrm{S}\right.$.). Smythe, Sabine and Elliot extended the analysis at St. Helena, and also determined the tide at Singapore. Several of the gifted directors of the Netherlands Government Observatory at Batavia $\left(6^{\circ} \mathrm{S}\right.$.) made valuable determinations there, as their barometric data accumulated.

These successes were possible because in the tropics the lunar atmospheric tide has its greatest amplitude, while the irregular variations there are less than elsewhere (except during extreme cyclonic conditions). Further attempts to determine the tide in temperate latitudes by Bouvard, Eisenlohr, Neumayer, Airy (using twenty years hourly observations at Greenwich), Börnstein, Morano and others proved unsuccess. ful, until 1918, when Chapman made a determination for Greenwich, where observations covering sixty-

* This article summarizes a Geophysical Discussion held at the Royal Astronomical Society on January 24, under the chairmanship of Si Edward Appleton. The Discussion, which was opened by Prof Sydney Chapman was continued by Sir Geoffrey Taylor, Dr. M. V. Wilke and Mr. $\mathbf{K}$. Weekes. 


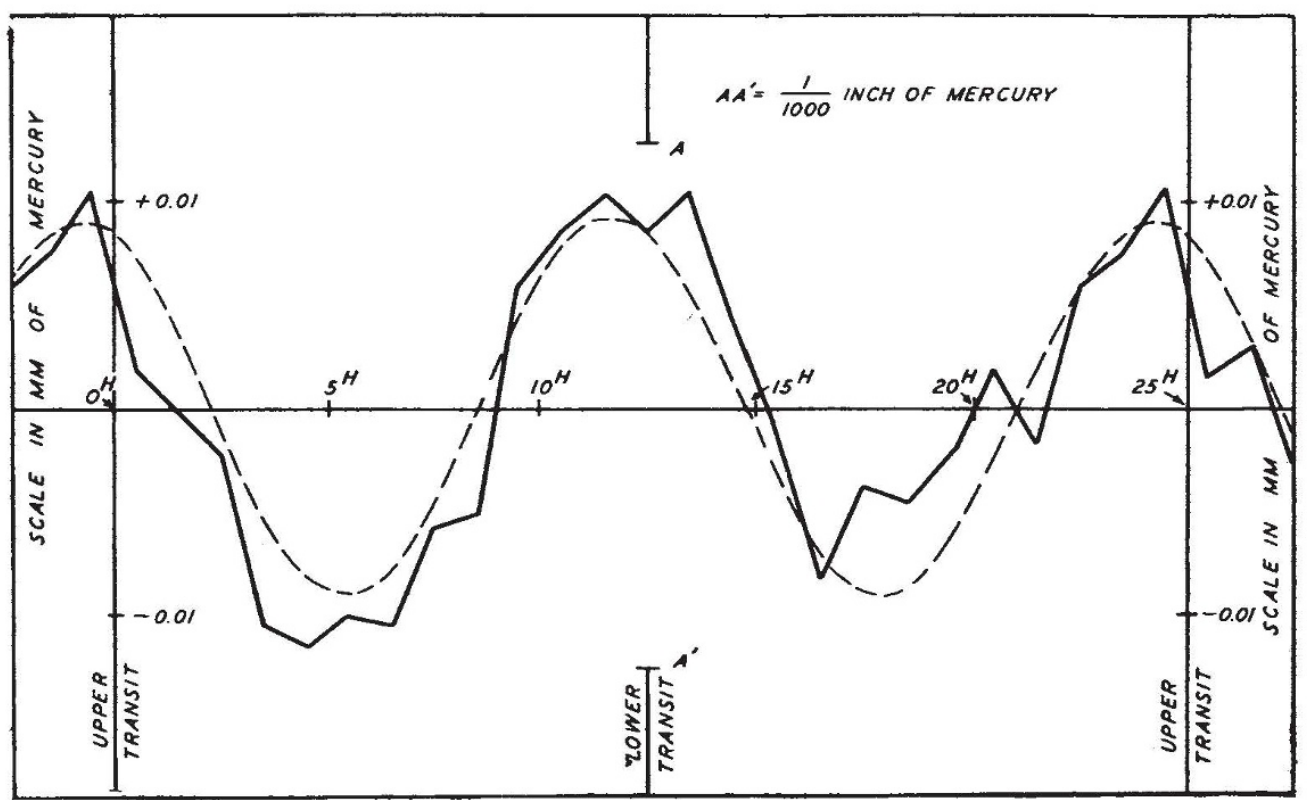

Fig. 1. THE LUNAR TIDAL BAROMFTRIC VARIATION AT GREFNWICH; - - - CURVE CALCULATED FROM THE HOURLY BAROMETRIC OBSERVATIONS ; - - , SEMI-DIURNAL COMPONENT OF THE CALOULATED CURVE

three years were by then available. Two thirds of the data were discarded, only those days being used on which the barometric range did not exceed $0.1 \mathrm{in}$. of mercury. Moreover, the last digit of the readings (made to 0.001 in.) was discarded. It was a remarkable illustration of the law of errors that the data, thus used only to $\mathbf{0 . 0 1}$ in., disclosed a lunar semidiurnal variation of pressure (Fig. 1) of total amplitude less than $\mathbf{0 . 0 0 1}$ in., the average change from hour to hour being of order 0.0001 in.

Since 1918 the number of determinations of the lunar atmospheric tide has greatly increased, its present value being about fifty-four (forty-four in the northern hemisphere, ten south of the equator; fifteen tropical, thirty-nine in higher latitudes). Bartels has obtained three of these, Pramanik also three, Robb and Tannahill one, most of the others having been derived by Chapman and his colleagues at the Imperial College of Science and Technology, where the speed of the work was greatly increased after 1930 by the generous loan of a set of Hollerith machines by the British Tabulating Machine Company, Ltd.

Some of the results are summarized in Fig. 2, which shows a number of arrows on a Mercator map of the world. Each arrow represents in length (on the scale indicated) the semi-amplitude of the lunar tidal variation of the barometer at the place situated at the centre of the arrow; if the arrow is regarded also as the hour hand of a clock that keeps lunar time (so that the northerly direction on the chart would correspond to $12^{\mathrm{h}}$ lunar, that is, upper or lower meridian passage of the moon), then the direction of the arrow indicates the time of maximum barometric pressure, or atmospheric high tide (lunar).

The chart shows that at most of the places considered the time of high tide is after lunar transit, by about $45 \mathrm{~min}$. in the northern hemisphere, and $20 \mathrm{~min}$. in the southern, where, however, there are large gaps in our knowledge ; but there are stations where the tide is certainly early, before the moon's passage, namely, Mauritius (No. 45 on the chart) and Kimberley (No. 46). The tide is greatest in the tropics, but the decrease with increasing latitude is not regular : places in the same latitude may have decidedly different air-tides; for example, Buenos Ayres (47) and Melbourne (48). These irregularities are perhaps surprising, as the atmospheric ocean is not complicated by lateral boundaries.

The lunar atmospheric tide seems to vary in amplitude from apogee to perigee, with the changing distance of the moon, in much the way that tidal theory would suggest. But it has an unexpected variation also throughout the year, which is not seasonal, as it does not change when the sun crosses the equator ; over most of the earth the tidal range is greatest at the June solstice; near the December solstice the lag after the moon is most pronounced, being more than half an hour later than the annual mean hour of tidal maximum. This change of the tide throughout the year still awaits an explanation.

\section{Solar Daily Oscillations}

Though in the oceans the daily changes of level are due primarily to the moon, in the atmosphere this is not so. The main periodic oscillation is solar semidiurnal, with maxima in barometric pressure at about 10 a.m. and 10 p.m. local time. This is shown clearly in tropical barograph records, of which it is the main feature, except when hurricanes pass the station. In temperate latitudes it is obscured by the far larger irregular changes of pressure, but it is easily determined by averaging the data of many days.

Though the phase of this 12-hour (solar) wave in the barometric variation is remarkably constant over the belt between $\pm 50^{\circ}$ latitude, with increasing latitude the oscillation tends to vary with absolute time, as first perceived by Greely. Through the work of Schmidt, Alt and Simpson, it has been shown that there are two solar semi-diurnal oscillations, one of tidal type, following the sun (and anti-sun) round the earth, the other being symmetrical about the earth's axis, and consisting of a 'zonal' oscillation of air between the equator and the poles. Simpson found that the corresponding two terms in the barometric variation are : 


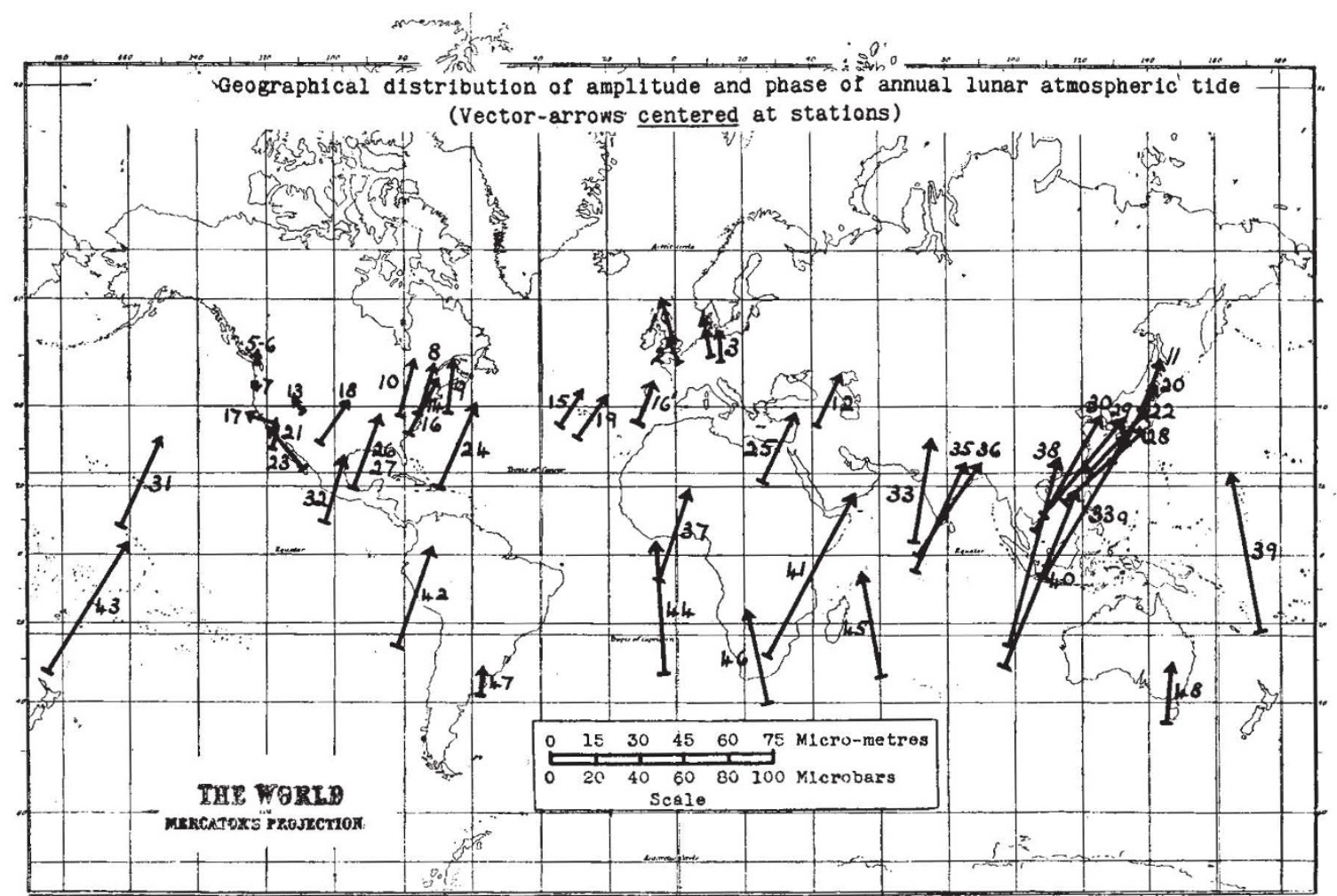

Fig. 2

$0.937 \cos ^{3} \varphi \sin \left(2 t+154^{\circ}\right) \mathrm{mm}$. of mercury and $0 \cdot 137\left(\sin ^{2} \varphi-\frac{1}{3}\right) \sin \left(2 t+105^{\circ}-2 \lambda\right) \mathrm{mm}$.,

where $\varphi$ denotes the latitude, $t$ the local mean solar time reckoned in angle at the rate $15^{\circ}$ per hour, and $\lambda$ denotes the longitude east of Greenwich. Thus at the equator the amplitude of the zonal part of the semi-diurnal oscillation is only $0.046 \mathrm{~mm}$.

The solar 12-hourly oscillation, like the lunar tide, has an annual variation, mainly not seasonal; in phase the change is opposite to that for the lunar tide, the maximum pressure occurring earliest in December and latest in July or August. The amplitude varies less than in the case of the lunar tide; at many places it has two maxima in the year, near the equinoxes, and two minima near the equinoxes.

In addition, there is a fairly regular world-wide oscillation of period 8 (solar) hours, represented approximately in the barometric variations by a term of amplitude $0.46 \sin \varphi \cos ^{3} \varphi \mathrm{mm}$. in December ; the phase is nearly opposite in the two hemispheres, and is nearly reversed from summer to winter.

\section{Theory of the Oscillations}

As Sir Geoffrey Taylor pointed out in the discussion, Laplace showed that in an isothermal atmosphere, oscillations involving no vertical motion, and in which the changes of volume occur isothermally, have the same free periods as those of corresponding mode (or geographical distribution) in a liquid ocean of a certain equivalent depth, which is actually the height of the equivalent homogeneous atmosphere. Margules developed the theory along these lines, stimulated by a hypothesis proposed by Kelvin, to explain why the solar semi-diurnal atmospheric oscillation so much exceeds the lunar (the ratio at the equator being about
15 to 1). Kelvin concluded that the former is of thermal origin, and that it is much magnified by resonance with a free period of atmospheric oscillation, of the same mode, very nearly approximating to 12 solar hours. Margules confirmed Kelvin's resonance hypothesis by finding a free period nearly coinciding with half a day. But this confirmation was unreliable, because the atmosphere and its oscillations are not isothermal, as Laplace's theory and Margules' calculations postulated.

Lamb investigated the horizontal propagation of plane waves in a non-rotating atmosphere on more realistic assumptions, taking into account vertical motion and adiabatic oscillations, but not the observed temperature-distribution with height. $\mathrm{He}$ then assumed that free oscillations of the atmosphere can exist which are identical in mode and period with those of an ocean of such depth that long waves are propagated in it with the speed that he calculated for plane waves. This assumption, which may be regarded as an extension of Laplace's theory of waves in an isothermal atmosphere, is not obviously true, since it involves the possibility that the atmosphere may have many equivalent depths, a contingency which is not possible in the case of an isothermal atmosphere. Lamb's assumption was later proved to be true by Taylor, who had used it previously to estimate the period to be expected in an atmosphere capable of carrying waves propagated at the speed observed in those produced by the Krakatoa volcanic eruption in 1883 ; Taylor concluded that the atmosphere has no free motion with a period so close to $\mathbf{1 2}$ hours as to fit Kelvin's resonance hypothesis. Later, however, he developed Lamb's work on oscillations having similar ground distributions but different height distributions of motion, 
taking account also of the cessation, at the tropopause, of the upward lapse of atmospheric temperature. Soon afterwards Pekeris applied Taylor's methods to determine the free periods of an atmosphere in which the stratospheric temperature again begins to increase upwards (as indicated by the abnormal propagation of sound to great distances) and then, still higher up, decreases once more. He showed that such an atmosphere could have a free oscillation of tidal type with a period very close to 12 hours, though the uncertainty of the upper atmospheric data as yet preclude the exact calculation of the period.

\section{Tides in the lonosphere}

The calculations by Pekeris indicated that the amplitude of his free oscillation of nearly half-day period would increase greatly with height. Soon afterwards, Appleton and Weekes announced the astounding but well-authenticated discovery of a lunar tidal twice-daily rise and fall of the $E$ layer of the ionosphere by nearly $\pm 1 \mathrm{~km}$., indicating an enormous magnification of the lunar tidal oscillation at high levels, as compared with the ground.

The study of the lunar daily geomagnetic variations had already shown the existence of the lunar tide in an electrically conducting layer of the atmosphere, reasonably to be identified with the ionosphere. The theory of these variations was in some ways rendered easier by the discovery of the large tide in the $E$-layer, which lessened the rather difficult demands made by the theory on the conductivity of the layer. The $E$-layer tide, however, was in phase with the moon, whereas the magnetic variations indicate an oscillation of opposite phase.

\section{New Calculations of the Forced Oscillations}

This interesting and complex situation as regards the facts and the theory of the atmospheric oscillations and their ionospheric effects calls both for further observations (in particular, as to the thermal structure of the atmosphere up to ionospheric levels) and renewed theoretical studies of the oscillations. Dr. M. V. Wilkes and Mr. K. Weekes described their new studies of this kind. The main input of tidal and thermal energy into the atmospheric oscillations is near the ground; energy passes upwards from thence, as if propagated in a medium with refractive index $\mu$ given by

$$
\mu^{2}=-\frac{1}{4}+\frac{1}{h}\left(\frac{\gamma-1}{\gamma} H+\frac{d H}{d x}\right),
$$

where $\gamma$ is the usual specific-heat ratio, $x$ is a co-ordinate depending on the height, $H$ the scale height $(R T / \bar{m} g)$ and $h$ the equivalent height of the ocean with the same free period for the type of oscillation considered. If the distribution of temperature $T$ (or $T / \bar{m}$, where $\bar{m}$ denotes the mean molecular weight) makes $\mu^{2}$ negative at a certain layer, this acts as a barrier against upward propaga. tion, like a total reflector, and resonance may occur. Using this method of approach, it is possible to see on general grounds that if the resonance theory is accepted the temperature in the atmosphere must be assumed to fall again (for example, to $200-240^{\circ} \mathrm{K}$.) above the first hot layer in the stratosphere. The boundary condition applied at high levels is that the flow of energy is upward. The energy is finally lost in the region above $150 \mathrm{~km}$., where the kinematic viscosity becomes so great as to cause heavy damping of the waves.
Mr. Weekes described the strenuous efforts made to find temperature-height distributions consistent with the high resonance of the solar half-daily oscillation at the ground, and of the lunar tidal oscillation in the ionosphere. Taking the stratospheric temperature to rise from $220^{\circ} \mathrm{K}$. to $400^{\circ} \mathrm{K}$. in the layer between 33 and $45 \mathrm{~km}$. height, and then to fall to $200^{\circ} \mathrm{K}$. (as suggested by the work of Ratcliffe, Budden and Wilkes on the propagation of long radio waves), the oscillation at about $100 \mathrm{~km}$. was found to be reversed, agreeing with the indications of the lunar tidal geomagnetic variation. This reversal of phase at high level, which was first found by Pekeris, is in contradiction to the experimental results of Appleton and Weekes. Further calculations have shown, however, that this discrepancy is not a fundamental one and can be resolved, for example, if the temperature is assumed to rise again to a further maximum in the $E$ region.

It may be expected that in the fairly near future the temperature-height distribution in the atmosphere will be actually measured by V 2 rocket methods. This will remove much that is now tentative in the theory of these atmospheric oscillations; but it remains to be seen whether the many pieces of evidence will then combine to form a clear coordinated picture, or whether discrepancies will persist and call for further search and effort.

\section{S. Chapman}

\section{HISTORY AND WORK OF THE PAL EONTOGRAPHICAL SOCIETY}

ARCH 23 marks the hundredth anniversary of 1 the foundation of the Palæontographical Society, the only Society which devotes all its resources to the illustration and description of British fossils. Members will celebrate this centenary of scientific work by holding the hundredth annual general meeting on the morning of Monday, March 31, when the present president, Prof. H. L. Hawkins, professor of geology in the University of Reading, will deliver a commemorative address ; in the afternoon, a public lecture will be delivered in the lecture room of the Geological Survey Museum, and in the evening, members will dine at the Hall of the Worshipful Company of Tallow Chandlers, Dowgate Hill, London.

Several years before the foundation of the Society, a group of seven enthusiasts had common interests in the study of Tertiary fossils, and acted together under the name of the London Clay Club; for the most part, their rich collections of fossils passed eventually to the British Museum (Natural History). The leading spirit of this club was J. S. Bowerbank (1797-1877), a well-to-do London distiller, keenly interested in many sciences, who in 1839 was one of the founders of the Royal Microscopical Society. The other members were F. E. Edwards (1799-1875), a solicitor; John Morris (1810-86), then a Kensing. ton pharmacist; J. de C. Sowerby (1787-1871), a naturalist who was continuing the illustration and publication of the "Mineral Conchology" started in 1812 by his father, J. Sowerby; N. T. Wetherell, M.R.C.S. $(1800-75)$, of Highgate; S. V. Wood (1798-1880), a retired officer of the Merchant Navy; and Alfred White. The members of this Club contributed papers to the learned societies and periodicals, and occasionally unique fossils from their collections 